CERN-TH/95-60

BRX TH-373

ULB-TH-95/04

\title{
Gauge Properties of Conserved Currents in Abelian Versus Nonabelian Theories
}

\author{
S. Deser ${ }^{1,2}$ and Marc Henneaux ${ }^{3,4}$ \\ ${ }^{1}$ Physics Department, Brandeis University, Waltham, MA 02254, USA \\ ${ }^{2}$ CERN, 1211 Geneva 23, Switzerland \\ ${ }^{3}$ Faculté des Sciences, Université Libre de Bruxelles, \\ Campus Plaine C.P. 231, B-1050 Bruxelles, Belgium \\ ${ }^{4}$ Centro de Estudios Científicos de Santiago, Casilla 16443, Santiago 9, Chile
}




\begin{abstract}
We clarify the physical origin of the difference between gauge properties of conserved currents in abelian and nonabelian theories. In the latter, but not in the former, such currents can always be written on shell as gauge invariants modulo identically conserved, superpotential, terms. For the "isotopic" vector and the stress tensor currents of spins 1 and 2 respectively, we explain this difference by the fact that the nonabelian theories are just the self-coupled versions of the abelian ones using these currents as sources. More precisely, we indicate how the self-coupling turns the non-invariantizable abelian conserved currents into (on-shell) superpotentials. The fate of other conserved currents is also discussed.
\end{abstract}




\section{Introduction}

There is a striking difference between the gauge behaviour of conserved currents in abelian and nonabelian theories. On the one hand, it has been recently established [1] for Yang-Mills theory that (on shell) all local conserved currents either are, or can be "improved" to become, gauge invariant through addition of an identically conserved superpotential; by contrast, gauge-variant conserved currents are present in the abelian context. On the other hand, it is known that one may reach the full nonabelian YM theory as well as Einstein gravity [2] and supergravity [3] precisely through making the respective original free abelian models self-interacting, with their abelian gauge-variant conserved currents as the sources. Our aim will be to follow the abelian to nonabelian transition to show that these two sets of results are in fact perfectly complementary.

Physically, one may distinguish two types of conserved currents: those that are themselves sources - including possibly of those fields out of which they are constructed - and others, such as the stress tensor in flat space physics, that are not the right-hand sides of any field equations, fhough their integrated charges may be physically important. There is a third category, identically conserved divergences of superpotentials, and we shall consider as equivalent two conserved currents that differ on-shell by an identically conserved one. We shall also say that a current is improvable if it is equivalent on-shell to an invariant one.

We will show here how the originally non-improvable currents of the free theories become improved when they become the (self-coupling) sources in the nonabelian regime. We will also show that all other currents lose their invariance and conservation in the transition.

\footnotetext{
${ }^{1}$ To this category also belong the infinitely many conserved "zilches" - like the BelRobinson tensor discussed below - peculiar to free field theories, but these seem not to be extensible to the nonabelian regime 四.
} 


\section{Vector Fields}

Consider first the abelian theory, a collection of free photons labelled by a colour index; for simplicity of notation, we use the $S U(2)$ case of three such vectors labelled by (colour)-vector notation, $\boldsymbol{A}_{\mu}$. The gauge-variant Noether current that follows from the invariance of the theory under rotations in internal space reads

$$
\boldsymbol{j}^{\mu}=\boldsymbol{F}_{a b e l}^{\mu \nu} \times \boldsymbol{A}_{\nu}, \boldsymbol{F}_{a b e l}^{\mu \nu}=\partial_{\mu} \boldsymbol{A}_{\nu}-\partial_{\nu} \boldsymbol{A}_{\mu}
$$

and is manifestly conserved on shell $\left(\partial_{\mu} \boldsymbol{F}_{a b e l}^{\mu \nu}=0\right)$. It is not improvable to be invariant under the abelian gauge transformations of the free theory (even on shell), being bilinear in the fields but involving only a single derivative. As indicated in [1], it is also the only non-improvable conserved current on a generic background spacetime.

Now, if we make $\boldsymbol{j}^{\mu}$ the source of its own field

$$
\partial_{\nu} \boldsymbol{F}^{\mu \nu}=\boldsymbol{j}^{\mu}
$$

(with the usual rescaling of the self-coupling constant) then antisymmetry of $\boldsymbol{F}^{\mu \nu}$ requires $\boldsymbol{j}^{\nu}$ to be conserved also on the new mass shell. A simple calculation [2] shows that this in turn requires the $\boldsymbol{F}^{\mu \nu}$ in (2) to be promoted to its full Yang-Mills form,

$$
\boldsymbol{F}^{\mu \nu}=\boldsymbol{F}_{y m}^{\mu \nu}=\boldsymbol{F}_{a b e l}^{\mu \nu}+\boldsymbol{A}_{\mu} \times \boldsymbol{A}_{\nu}
$$

so that (2) become the usual Yang-Mills equations $\boldsymbol{D}_{\mu} \boldsymbol{F}_{y m}^{\mu \nu}=\left(\partial_{\mu}+\boldsymbol{A}_{\mu} \times\right)$ $\boldsymbol{F}_{y m}^{\mu \nu}=0$. The abelian current in (11) is correspondingly promoted to be a conserved gauge invariant object under the non-abelian deformation of the original abelian transformation. This promotion is formally trivial; just write $\AA^{2}$

$$
\boldsymbol{j}^{\mu}=\boldsymbol{F}_{y m}^{\mu \nu} \times \boldsymbol{A}_{\nu}=-\boldsymbol{D}_{\nu} \boldsymbol{F}_{y m}^{\mu \nu}+\partial_{\nu} \boldsymbol{F}_{y m}^{\mu \nu},
$$

to exhibit that the current is the sum of a superpotential and a gauge covariant term; the latter term actually vanishes on-shell, in agreement with the general theorem of [1] according to which a conserved current in YangMills theory can always be redefined to be gauge invariant by the addition

\footnotetext{
${ }^{2}$ One could also define a seemingly different $\boldsymbol{J}^{\mu}$ as the right side of $\partial_{\mu} \boldsymbol{F}_{a b e l}^{\mu \nu}=\boldsymbol{J}^{\nu}$, but it merely differs from $\boldsymbol{j}^{\mu}$ by the superpotential $\partial_{\mu}\left(\boldsymbol{A}_{\mu} \times \boldsymbol{A}_{\nu}\right)$.
} 
of equation of motion and superpotential terms. In the abelian limit, the right-hand side of (4) would of course identically vanish, reminding us of the non-improvability there. [Note also that $\boldsymbol{j}^{\mu}$ is not covariantly conserved, due to the superpotential in (4).] Thus we see that the consistent self-coupling of massless vector fields provides a mechanism by which the non-improvable current (1) of the abelian theory becomes improved and actually equivalent to an identically conserved current. This follows from the precise form of the interaction, which promotes the originally non-improvable current to be the source of the divergence of the antisymmetric tensor $\boldsymbol{F}_{y m}^{\mu \nu}$. Differently put, the current $\boldsymbol{j}^{\mu}$ is improvable as soon as there is a shell for it to be on. This shell is given by the consistent self-coupling, which eliminates all the non-improvable currents.

The abelian theory also contained one (and only one) conserved (and invariant) tensor current, namely the field strength $\boldsymbol{F}_{a b e l}^{\mu \nu}$ itself [5]. Corresponding to the fact that $\boldsymbol{F}^{\mu \nu}$ becomes covariant in the nonabelian case, it also becomes covariantly conserved, so that there remain no ordinarily conserved higher rank quantities either.

For completeness, we consider finally the rather different, identically conserved currents such as the abelian

$$
\tilde{\boldsymbol{J}}_{a b e l}^{\mu}=\boldsymbol{A}_{\nu} \times \tilde{\boldsymbol{F}}_{a b e l}^{\mu \nu}, \equiv \frac{1}{2} \partial_{\alpha} \epsilon^{\mu \nu \alpha \beta} \boldsymbol{A}_{\nu} \times \boldsymbol{A}_{\beta} ; \quad \tilde{\boldsymbol{F}}_{a b e l}^{\mu \nu} \equiv \frac{1}{2} \epsilon^{\mu \nu \alpha \beta} \boldsymbol{F}_{\alpha \beta}^{a b e l}
$$

Although the non-abelian generalization does not promote $\tilde{\boldsymbol{\jmath}}_{a b e l}^{\mu}$ to be a source current, $\tilde{\boldsymbol{J}}_{\text {abel }}^{\mu}$ remains identically conserved nevertheless if (as was done for $\boldsymbol{j}^{\mu}$ above) $\tilde{\boldsymbol{F}}_{\text {abel }}^{\mu \nu}$ is replaced by $\tilde{\boldsymbol{F}}_{y m}^{\mu \nu}$, as is easily verified using the Bianchi identity $\boldsymbol{D}_{\mu} \tilde{\boldsymbol{F}}_{y m}^{\mu \nu} \equiv 0$. Indeed, here too we can write the superpotential form

$$
\tilde{\boldsymbol{\jmath}}_{y m}^{\nu}=0+\partial_{\mu} \tilde{\boldsymbol{F}}_{y m}^{\mu \nu}
$$

as an identity, so although $\tilde{\boldsymbol{\jmath}}_{y m}^{\mu}=\tilde{\boldsymbol{\jmath}}_{\text {abel }}^{\mu}$, it is nonabelian gauge invariant (namely zero) up to a superpotential, quite independently of any Yang-Mills dynamics.

In $\mathrm{d}=3$, one may also consider the Chern-Simons action $I=\int d^{3} x \boldsymbol{A}_{\mu}$. $\tilde{\boldsymbol{F}}_{\text {abel }}^{\mu}$; the corresponding abelian current is the "descent" of (5) to $d=3$, namely $\boldsymbol{j}_{\text {abel }}^{\mu}=\epsilon^{\mu \alpha \beta} \boldsymbol{A}_{\alpha} \times \boldsymbol{A}_{\beta}$, conserved by virtue of the field equations $\tilde{\boldsymbol{F}}_{\text {abel }}^{\mu}=0$. Unlike (5), this $\boldsymbol{j}^{\mu} \underline{\text { not }}$ identically conserved and so can be set as a source of $\tilde{\boldsymbol{F}}^{\mu}$. The resulting non-abelian field equation, $\boldsymbol{F}_{y m}^{\mu \nu}=0$, implies 
that $\boldsymbol{j}^{\mu}$ is an on-shell gauge invariant object,

$$
\boldsymbol{j}^{\mu}=\tilde{\boldsymbol{F}}^{\mu}+2 \partial_{\alpha} \epsilon^{\mu \alpha \beta} \boldsymbol{A}_{\beta}
$$

just as in (雨).

\section{Tensor Fields}

The spin 2 situation is so similar to that for spin 1 that we omit all details (see $[2])$. The analog of the iso-current (1) is the stress tensor $T^{\mu \nu}$ of the free field action, which is conserved but neither gauge invariant nor improvable, being of second derivative order, while invariance would require it to be bilinear in the (linearized) curvature and hence of fourth order. When $T^{\mu \nu}$ is made the source of the linearized Einstein equations, thereby defining the full Einstein theory, it remains (ordinarily, not covariantly) conserved - on full mass shell - and can be expressed, in parallel with (四), as

$$
T^{\mu \nu}=-G^{\mu \nu}+G_{L}^{\mu \nu}
$$

where $G^{\mu \nu}$ is the full covariant Einstein tensor and its linearization is of course the divergence of a superpotential, $G_{L}^{\mu \nu}=\partial_{\lambda} S^{\lambda \mu \nu}$. Thus $T^{\mu \nu}$ is itself deformed gauge (diffeomorphism) invariant on shell up to that superpotential.

In the vector case, we noted the existence of the linear tensor current, $\boldsymbol{F}^{\mu \nu}$, conserved (only) at abelian level. There are actually two different higher rank analogs here. The first is linear in the fields, like $\boldsymbol{F}^{\mu \nu}$ : it is the set of superpotentials $S^{\lambda \mu \nu}$ of $G_{L}^{\mu \nu}$. They are conserved on linear Einstein shell, but (unlike $\boldsymbol{F}^{\mu \nu}$ ) gauge-variant; their conservation (like that of $\boldsymbol{F}^{\mu \nu}$ ) does not survive self-coupling [5]. The second higher rank quantity is more akin to the Maxwell stress-tensor, being bilinear in the fields: The abelian spin 2 theory allows for the existence of the gauge-invariant, conserved, totally symmetric and traceless (in $\mathrm{d}=4$ ), Bel-Robinson tensor.[

$$
B_{\alpha \beta \gamma \delta}=R_{\alpha \rho \gamma \sigma} R_{\beta}{ }^{\rho}{ }^{\sigma}+\tilde{R}_{\alpha \rho \gamma \sigma} \tilde{R}_{\beta}{ }^{\rho}{ }^{\sigma}{ }^{\sigma},
$$

where $\tilde{R}$ is the (left pair) dual. [Compare the vector field's stress tensor, $\left.2 T_{\mu \nu}=\boldsymbol{F}_{\mu}{ }^{\alpha} \cdot \boldsymbol{F}_{\nu \alpha}+\tilde{\boldsymbol{F}}_{\mu}{ }^{\alpha} \cdot \tilde{\boldsymbol{F}}_{\nu \alpha}.\right]$ Just as $\boldsymbol{F}_{\mu \nu}$ does, $B_{\alpha \beta \gamma \delta}$ becomes gaugecovariant and covariantly conserved in the full theory; there are no ordinarily conserved diffeomorphism-invariant local quantities in curved space.

\footnotetext{
${ }^{3}$ This fourth-rank tensor cannot, however, be made the source of any gravitational quantity nor even of a spin 4 field [7].
} 


\section{Comments}

The two examples we have exhibited of gauge-variant current improvement through the mechanism of self-coupling are essentially unique. [The only other case is the construction of supergravity [3] using the simultaneous gravitational stress tensor self coupling and that of the spin 3/2-spin 2 supercurrent.] Gauge fields of higher spin just do not possess local conserved free-field currents of sufficiently high rank to replicate the constructions given above. Spin 3 provides the simplest example of this failure [8]. Of course, using a conserved abelian current is not the only consistent way to self-couple a gauge system, but it is the only physically interesting one [9].

In itself, the existence of gauge variant conserved currents in abelian theories is not worrisome, so long as they are not physical sources. For example, as we have noted, the stress tensors of any spin $>2$ gauge fields are non-improvable, but they do obey the one physical requirement that their integrated charges - the Poincare generators - be gauge invariant [6]. Indeed, those free fields - (abelian) N-forms - that do have invariant stress tensors, instead encounter a clash between conservation and gauge invariance (in all but one critical dimension) when it comes to the conformal group [10]. There is no local Lorentz covariant expression for even the dilation current that respects gauge invariance, as witness the unimprovable expression [1] for Maxwell theory,

$$
D^{\mu}=x^{\nu} T_{\nu}^{\mu}+\frac{1}{2}(d-4) F_{a b e l}^{\mu \nu} A_{\nu} .
$$

Expressions similar to (10) hold for arbitrary N-form fields [10] in dimension other than $2(N+1)$, and indeed conformal (but of course not Poincaré!) charges lose their significance as a result.

In summary, we have provided a physical explanation of the findings of [1, 5] about the existence of non-improvable gauge variant currents in free spin 1 and 2 gauge theories and their absence when consistent self-interactions are turned on. In particular, we saw that those currents that can be turned into self-sources thereby acquire the - deformed - gauge covariance of the resulting nonlinear theory, modulo superpotentials. [Identically conserved currents behave similarly without use of dynamics.] For higher spins there is no selfcoupling mechanism available, and even N-form abelian fields have "bad" conserved currents that cannot generally be improved in a local fashion. 


\section{Acknowledgements}

M.H. is grateful to Glenn Barnich and Friedemann Brandt for useful conversations. This work has been supported in part by NSF grant PHY-9315811, by research contracts with the Commission of the European Communities and by research funds from the Fonds National de la Recherche Scientifique (Belgium). 


\section{References}

[1] G. Barnich, F. Brandt and M. Henneaux, Phys. Lett. B (in press).

[2] S. Deser, Gen. Rel. Grav. 1 (1970) 9; Class. Quant. Grav. 4 (1987) L99.

[3] D.G. Boulware, S. Deser, and J.H. Kay, Physics 96A (1979) 141.

[4] S. Deser and H. Nicolai, Phys. Lett. 98B (1981) 45.

[5] G. Barnich, F. Brandt and M. Henneaux, Commun. Math. Phys. (in press)

[6] S. Deser and J. Mc Carthy, Class. Quant. Grav. 7 (1990) L119.

[7] S. Deser and Z. Yang, Class. Quant. Grav. 7 (1990) 1491.

[8] F.A. Berends, G.H. Burgers and H. Van Dam, Nucl. Phys. B 260 (1985) 295; A.K.H. Bengtsson, Phys. Rev. D 32 (1985) 2031; T. Damour and S. Deser, Class. Quant. Grav. 4 (1987) L95.

[9] R. Wald, Phys. Rev. D33 (1986) 3613.

[10] S. Deser and A. Schwimmer, Int. J. Math. Phys. B8 (1994) 3741. 